\title{
Trabeculectomy ab interno (trabectome): yet another possibility in the treatment of uncontrolled glaucomatocyclitic crisis under systemic valganciclovir therapy?
}

M Pahlitzsch ${ }^{1}$, N Torun ${ }^{1}$, J Gonnermann, A-KB Maier, U Pleyer, E Bertelmann, A Joussen and MKJ Klamann

\begin{abstract}
Purpose To assess the outcome of trabectome surgery in the treatment of glaucomatocyclitic crisis (Posner-Schlossman syndrome) in patients with uncontrolled intraocular pressure (IOP).

Patients/Methods Trabectome surgery was performed in seven patients with diagnosed glaucomatocyclitic crisis and uncontrolled IOP where cytomegalovirus DNA was verified by polymerase chain reaction in aqueous humour samples. All patients were treated with oral valganciclovir. After surgery the patients were followed-up for 12 months. Results Mean IOP before trabectome surgery was $40 \pm 10 \mathrm{~mm} \mathrm{Hg}$ (range $33-58 \mathrm{~mm} \mathrm{Hg}$ ). The mean number of antiglaucoma medication prior to surgery was $3.1 \pm 0.4$. By the end of the 12 months, IOP in all patients was reduced to normal level $(13 \pm 1 \mathrm{~mm} \mathrm{Hg})$ and their antiglaucoma medication was decreased to $0.8 \pm 1.1$. No recurring attack of glaucomatocyclitic crisis occurred.
\end{abstract}

Discussion In addition to oral valganciclovir therapy, trabectome surgery seems to be a reliable and effective tool for the management of glaucomatocyclitic crisis with uncontrolled IOP.

Eye (2015) 29, 1335-1339; doi:10.1038/eye.2015.112; published online 3 July 2015

\section{Introduction}

The Posner-Schlossman syndrome (PSS; glaucomatocyclitic crisis) was first described by Posner and Schlossmann in 1948: ${ }^{1}$ Its criteria included recurring unilateral episodes of elevated intraocular pressure (IOP), mild anterior uveitis, sectoral iris atrophy, and keratic precipitates (fine, unpigmented precipitates). ${ }^{2,3}$ In only $30-40 \%$ of the cases obvious differences in color or heterochromia are present, whereas the hypochromic dystrophy of the iris was dependent on the magnitude and duration of the cyclitic process. ${ }^{3}$

In the pathogenesis of PSS, the cytomegalovirus out of the large Herpes virus group was detected as the most important factor driving the inflammation process. ${ }^{4-7}$

Samples of aqueous humour could be used to verify CMV DNA by polymerase chain reaction (PCR) in PSS patients. ${ }^{8}$ Therefore systemic and topical antiviral therapy was recommended in these subjects. ${ }^{2}$ Because topical treatment could not prevent the recurrence, intravitreal injections of valganciclovir were carried out to decrease the recurrence rate. ${ }^{9-11}$ It could be demonstrated that ester prodrugs generated therapeutic intravitreal concentrations of ganciclovir in vivo and the mean residence time of ganciclovir could be enhanced by three to fourfold through prodrug modification. ${ }^{12}$ Patients controlled only by topical antiglaucoma and antiviral therapy showed high numbers of frequent recurrences, so oral valganciclovir therapy was established. Chee $e$ t $a l^{9}$ suggested a 3-month course of oral valganciclovir (response rate 90.9\%). However, the recurrence rate after cessation of this therapy was very high ( $80 \%)$. Moreover, $100 \%$ of patients in the group treated with intravitreal ganciclovir had a recurrence. ${ }^{9}$ Extented studies about the duration of oral valganciclovir therapy were reported by Sobolewska et al. ${ }^{13}$ In long-term follow-up a response rate of $63.6 \%$ could be demonstrated (7 of 11 patients); oral
Department of ophthalmology, Campus Virchow clinic, Charite University medicine, Berlin, Germany

Correspondence: M Pahlitzsch, Department of ophthalmology, Campus Virchow clinic, Charite University medicine, Augustenburger Platz 1, Berlin 13353, Germany Tel: +49 304506 54325; Fax: +49 30450554944 E-mail: milena.pahlitzsch@ charite.de

${ }^{1}$ These authors contributed equally to this work.

Received: 19 November 2014

Accepted in revised form:

31 May 2015

Published online:

3 July 2015 
valganciclovir therapy led to a resolution of inflammatory activity and stable IOP. Nevertheless, despite adequate systemic valganciclovir treatment IOP decompensation is still a problem in PSS. ${ }^{14} \mathrm{Ab}$ interno trabeculectomy (trabectome) can be offered as a minimally invasive device for the surgical treatment of open-angle glaucoma, and as an alternative method for highly complicated and complex open-angle glaucoma surgery. ${ }^{15-17}$ As the criteria of PSS included an open angle in the gonioscopy, this patient cohort may be suitable for trabectome surgery to decrease the IOP. ${ }^{3}$

The aim of the present study was to assess the outcome of trabectome surgery in the treatment of glaucomatocyclitic crisis in patients with uncontrolled IOP.

\section{Patients and methods}

This retrospective cohort outcome study included seven patients with PSS and uncontrolled IOP. The patients fulfilled the clinical criteria of PSS (recurrent unilateral episodes of elevated IOP, mild anterior uveitis, sectoral iris atrophy and fine, and unpigmented keratic precipitates). Furthermore, cytomegalovirus DNA was verified by PCR in aqueous humour samples. The patients were treated with oral valganciclovir $(2 \times 900 \mathrm{mg}$ twice daily for 3 weeks), topical corticosteroid therapy (Inflanefran forte eye drops five times daily) and local antiglaucoma medication. This study followed the ethical standards of the Declaration of Helsinki principles. Inclusion criteria beside diagnosed PSS, as described above, were uncontrolled IOP elevation ( $>30 \mathrm{~mm} \mathrm{Hg}$ ) with insufficient local antiglaucoma medication. Patients with a 2-week period of uncontrolled IOP were included. Patients were excluded if they did not reach a minimum follow-up of 12 months after trabectome surgery, no cytomegalovirus DNA was verified by PCR in aqueous humour samples, or if patients had any previous eye surgery.

Each patient underwent a complete ophthalmological examination, including a medical history review, bestcorrected visual acuity measurement, slit-lamp biomicroscopy, Goldmann's applanation tonometry, gonioscopy, dilated fundus examination including the Jonas criteria, and a baseline bilateral standard automated perimetry threshold visual field test using the 24-2 Swedish interactive threshold algorithm (Humphrey Visual Field Analyzer, Carl Zeiss Meditec, Dublin, CA, USA). ${ }^{18}$ Examinations were performed prior to surgery, 1 day, 6 weeks, 3 months, 6 months, and 12 months after surgery. IOP readings and the number of antiglaucoma medication was evaluated at every follow-up visit.

\section{Surgical technique}

After informed consent was provided, an anterior chamber tap (AC tap) was performed in all patients during activated inflammatory situation. The aqueous humour was analysed for CMV using PCR.

The trabectome surgery was performed by one of two experienced surgeons (MKJK, NT) using the same surgical protocol under local (peribulbar) anesthesia.

Briefly, a $1.6 \mathrm{~mm}$ near limbal temporal corneal incision was made. Under gonioscopic view the trabectome handpiece was advanced nasally and inserted through the trabecular meshwork. Selective electrosurgical ablation was activated to remove an $\sim 120$-degree arc of trabecular meshwork and inner wall of the Schlemm canal, while protecting the surrounding tissue and collector channels with the ceramic-coated footplate.

Postoperative treatment was comprised of a topical combination of steroids and antibiotics.

In addition, pilocarpin 1\% eye drops 4 times per day were slowly reduced over 4 weeks after surgery to avoid anterior synechiae. Antiglaucoma medication was continued as needed. After surgery, ongoing valganciclovir therapy (450 mg twice daily) was set up for 3 months in each patient.

\section{Statistical analysis}

The assessment of all statistical data was calculated by SPSS (SPSS version 19.0, SPSS, New York, NY, USA). Preoperative and postoperative values were compared with the independent sample $t$-test. Numeric variables that were not normally distributed were compared with the Mann-Whitney $U$-test. Scatterplots of the individual IOP trend were designed. A $P$-value of $<0.05$ was considered as indicating a statistically significant difference.

\section{Results}

Seven patients ( 3 males, 4 females, mean age $44.2 \pm 14.1$ years) were included in this study from July 2013 to January 2014. All seven patients were phakic. The demographic data of this study population were summarized in Table 1.

Mean IOP before ab interno trabeculectomy was $40 \pm 10 \mathrm{~mm} \mathrm{Hg}$ (range 33-58 $\mathrm{mm} \mathrm{Hg}$ ). The mean IOP postoperatively was $11 \pm 4 \mathrm{~mm} \mathrm{Hg}, 12 \pm 3 \mathrm{~mm} \mathrm{Hg}$, $12 \pm 2 \mathrm{~mm} \mathrm{Hg}$, and $12 \pm 2 \mathrm{~mm} \mathrm{Hg}$ after 1 day, 6 weeks, 3 months, and 6 months, respectively. Mean IOP was stabilised at $13 \pm 1 \mathrm{~mm} \mathrm{Hg}$ at 1-year follow-up. In Figure 1, the trend of the intraocular pressure was analysed for each individual preoperative compared with postoperative follow-up controls. 
Table 1 Demographic and clinical characteristics of the study cohort

\begin{tabular}{lccccrr}
\hline No. & Gender & $\begin{array}{c}\text { Age } \\
\text { (years) }\end{array}$ & Eye & $\begin{array}{c}\text { IOP preOp } \\
(\mathrm{mm} \mathrm{Hg})\end{array}$ & MD & PSD \\
\hline 1 & Male & 40 & Right & 40 & -4.2 & -3.8 \\
2 & Female & 70 & Left & 30 & -3.7 & -2.4 \\
3 & Female & 39 & Right & 30 & 4.5 & 3.1 \\
4 & Female & 53 & Right & 33 & 5.7 & 4.2 \\
5 & Male & 55 & Left & 58 & 3.4 & 1.8 \\
6 & Male & 25 & Left & 42 & 6.1 & 5.3 \\
7 & Female & 48 & Right & 48 & -4.8 & -2.9 \\
\hline
\end{tabular}

Abbreviations: MD, mean deviation; PSD, pattern standard deviation of the visual field examination.

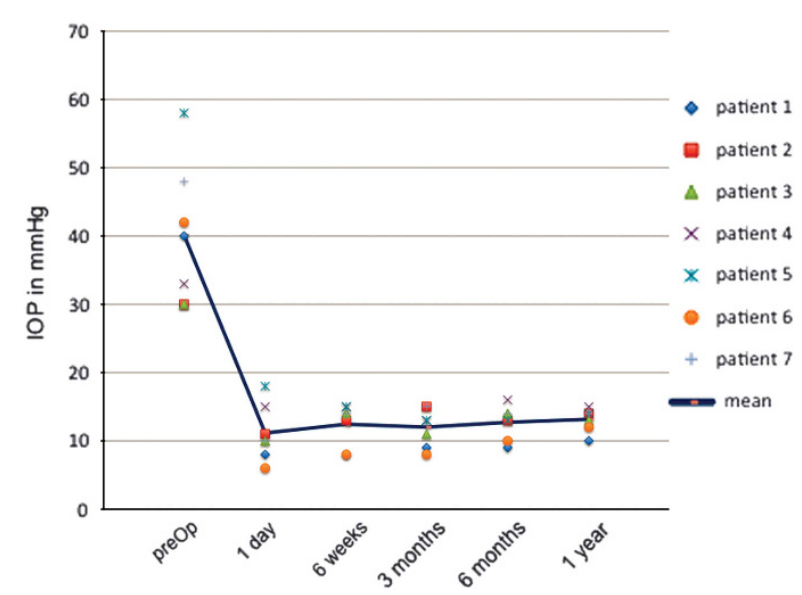

Figure 1 Analysis of the IOP trend in all seven individuals comparing the preoperative to postoperative follow-ups.

The mean number of antiglaucoma medication prior to surgery was $3.1 \pm 0.4$. Glaucoma medication decreased to $2.1 \pm 1.0$ ( 1 day postoperative), $1.4 \pm 1.1$ (6 weeks postoperative), $0.8 \pm 1.0$ ( 3 months postoperative), and $0.8 \pm 1.0$ (6 months postoperative). After 1 year of follow-up the mean number of medication was still reduced to $0.8 \pm 1.1$.

The most common complication was intraoperative blood reflux, which occurred in all eyes. There was no incidence of 1-day postoperative hypotony or IOP spikes. No incidences of sustained hypotony, choroidal effusion or hemorrhage, visual acuity decrease, infection, aqueous misdirection, or wound leak were noted.

\section{Discussion}

After the cytomegalovirus was detected as the main pathogenetic mechanism of the PSS it was interesting to discover whether the anterior segment inflammation and the number of cytomegalovirus copies in the aqueous humour were correlated with the disease characteristics. Kandori et al ${ }^{19}$ demonstrated that the number of cytomegalovirus copies was significantly correlated with the number of recurrent episodes and IOP elevation, but was not significantly correlated with the disease type. These results were confirmed by Miyanaga et al, ${ }^{20}$ a significant corneal endothelium cell loss was recorded with high viral loads of CMV in the aqueous humour. In the treatment of PSS positively tested for cytomegalovirus in the aqueous humour, Chee et al ${ }^{9}$ recommended a 3-month course of oral valganciclovir therapy (response rate $90.9 \%){ }^{7}$ On the basis of a high recurrence rate of $80 \%$ after cessation of the therapy, long-term studies followed evaluating the advised time of duration of oral valganciclovir therapy. Sobolewska et al ${ }^{13}$ demonstrated a response rate of $63.6 \%$ (7 of 11 patients); in 6 patients, the therapy could be discontinued after a mean of 14 months. However, in some cases IOP could not be reduced to normal levels. In our study, all patients had positive cytomegalovirus DNA in anterior chamber taps and were treated with oral valganciclovir as described above. Yet in this selected PSS cohort, patients had uncontrolled IOP elevation (>30 mm Hg) with insufficient local antiglaucoma medication. The present study examined the outcome of trabectome surgery in the treatment of PSS in patients with uncontrolled IOP.

Previous studies showed, that trabectome surgery led to an average decrease of IOP between $6-7 \mathrm{~mm} \mathrm{Hg}$ in open-angle glaucoma, ${ }^{21-23}$ and $7-12 \mathrm{~mm} \mathrm{Hg}$ in exfoliation glaucoma. ${ }^{22,23}$ In open-angle glaucoma the mean number of medication could be reduced from $2.1 \pm 1.3$ to $1.2 \pm 1.1 .^{21}$ In addition, in the PEX cohort antiglaucoma medication was reduced from $2.0 \pm 1.2$ to $1.1 \pm 1.1 .^{22}$ In our PSS study a strong statistically significant reduction of IOP from $40 \pm 10 \mathrm{~mm} \mathrm{Hg}$ to $13 \pm 1 \mathrm{~mm} \mathrm{Hg}$ could be achieved by trabectome surgery in this PSS cohort in 1 year. At the 1-day follow-up the mean IOP was already $11 \mathrm{~mm} \mathrm{Hg} \pm 4 \mathrm{~mm} \mathrm{Hg}$. The antiglaucoma topical medication was decreased from $3.1 \pm 0.4$ preoperative to $0.8 \pm 1.1$ at 12 -months follow-up.

The most common complication after trabectome surgery was intraoperative blood reflux (in seven out of seven patients), which occurred when removing an arc of trabecular meshwork and an inner wall of Schlemm canal. ${ }^{24}$ This indicated a reflux from collector channels communicating with the episcleral venous systems. ${ }^{23}$ Another complication described in the literature was post-trabectome IOP elevation which was due to the cleft closure induced by membrane growth and/or peripheral anterior synechiae. ${ }^{23}$ Wang et al ${ }^{25}$ demonstrated that $\mathrm{Nd}$ : YAG goniopuncture was an effective tool in closure lysis and in normalizing the IOP back to its pre-trabectome level. However, no postoperative IOP elevation was described in our patients with PSS.

The occurrence of postoperative complications typically associated with filtering procedures such as hypotony, wound leak, flat anterior chamber, choroidal effusion, 
hemorrhage, hyphema and infection were not reported in ab interno trabeculectomy. ${ }^{15-17}$ However filtration glaucoma surgery still led to a more intense lowering of the IOP.

Zhong et al ${ }^{14}$ performed trabeculectomy surgery in a PSS cohort ( 2 males, 6 females) with uncontrolled IOP $(50 \pm 5 \mathrm{~mm} \mathrm{Hg})$ and visual field defects and stated it as an effective method to decrease IOP to normal level $(14 \pm 2 \mathrm{~mm} \mathrm{Hg})$ without any antiglaucoma medications. Compared with our study, the IOP after trabectome surgery decreased significantly as well. In contrast to Zhong et al, ${ }^{14}$ the glaucoma medication after trabectome surgery was tapered down but needed to be continued after 12 months. However, the lack of ocular surface alterations remains as the advantage of trabectome surgery ${ }^{22}$ and its attempt to increase outflow facility along the natural pathway. ${ }^{23,26}$

There were limitations of this study that must be addressed: The study had a limited number of patients and a short follow-up period for glaucoma. However, this is the first study focusing on trabectome surgery in this specific patient cohort.

In conclusion, in addition to the oral valganciclovir therapy trabectome surgery seems to be a reliable and effective tool for the management of glaucomatocyclitic crisis with uncontrolled IOP.

\section{Summary}

\section{What was known before}

- The glaucomatocyclitic crisis and uncontrolled intraocular pressure (IOP) were associated with the cytomegalovirus (CMV) infection; CMV DNA was verified by polymerase chain reaction in aqueous humour samples in different comparative studies. For treating the intraocular inflammation reaction and lowering the IOP, oral valganciclovir therapy was already known.

\section{What this study adds}

- Decompensation of IOP in the Posner-Schlossmann syndrome with patients already taking high-dose valgancivlovir therapy can be safely treated by trabectome surgery. This intervention leads to a significant decrease of the IOP after 1 year of follow-up, without inducing a recurrent inflammation.

\section{Conflict of interest}

The authors declare no conflict of interest.

\section{References}

1 Posner A, Schlossman A. Syndrome of unilateral attacks of glaucoma with cyclitic symptoms. Arch Ophthalmol 1948; 39: 517-535.
2 de Schryer I, Rozenberg F, Cassoux N, Michelson S, Kestelyn P, Lehoang P et al. Diagnosis and treatment of cytomegalovirus iridocyclitis without retinal necrosis. $\mathrm{Br} \mathrm{J}$ Ophthalmol 2006; 90: 852-855.

3 Hollwich F. Clinical aspects and therapy of the PosnerSchlossmann-syndrom. Klin Monbl Augenheilkd 1978; 172(5): 736-744.

4 Bloch-Michel E, Dussaix E, Cerqueti P, Patarin D. Possible role of cytomegalovirus infection in the etiology of the Posner-Schlossman syndrome. Int Ophthalmol 1987; 11: 95-96.

5 Teoh SB, Thean L, Koay E. Cytomegalovirus in etiology of Posner-Schlossman syndrome: evidence from quantitative polymerase chain reaction. Eye 2005; 19: 1338-1340.

6 Chung RS, Chua CN. Intravitreal ganciclovir injections in aqueous cytomegalovirus DNA positive hypertensive iritis. Eye 2006; 20: 1080.

7 Yamamoto S, Pavan-Langston D, Tada R, Yamamoto R, Kinoshita S, Nishida K et al. Possible role of herpes simplex virus in the origin of Posner-Schlossman syndrome. Am J Ophthalmol 1995; 119(6): 796-798.

8 Yamamoto S, Pavan-Langston D, Kinoshita S, Nishida K, Shimomura Y, Tano Y. Detecting herpesvirus DNA in uveitis using the polymerase chain reaction. Br J Ophthalmol 1996; 80 (5): 465-468.

9 Chee SP, Jap A. Cytomegalovirus anterior uveitis: outcome of treatment. Br J Ophthalmol 2010; 94: 1648-1652.

10 Hwang YS, Link KK, Lee JS, Chang SH, Chen KJ, Lai CC et al. Intravitreal loading injection of ganciclovir with or without adjunctive oral valganciclovir for cytomegalovirus anterior uveitis. Graefes Arch Clin Exp Ophthalmol 2010; 248: 263-269.

11 Morlet N, Young S, Naidoo D, Graham G, Coroneo MT. High dose intravitreal ganciclovir injection provides a prolonged therapeutic intraocular concentration. $\mathrm{Br} J$ Ophthalmol 1996; 80: 214-216.

12 Macha A, Mitra AK. Ocular disposition of ganciclovir and its monoester prodrugs following intravitreal administration using microdialysis. Drug Metab Dispos 2002; 30: 670-675.

13 Sobolewska B, Deuter C, Doycheva D, Zierhut M. Long-term oral therapy with valganciclovir in patients with Posner-Schlossman syndrome. Graefes Arch Clin Exp Ophthalmol 2013; 252(1): 117-124.

14 Zhong Y, Cheng Y, Liu X, Feng P. Trabeculectomy in the management of glaucomatocyclitic crisis with visual field defect. Ocul Immunol Inflamm 2010; 18(3): 233-236.

15 Jea SY, Francis BA, Vakili G, Filippopoulos T, Rhee DJ. $\mathrm{Ab}$ interno trabeculectomy versus trabeculectomy for openangle glaucoma. Ophthalmology 2012; 119: 36-42.

16 Jampel HD, Musch DC, Gillespie BW, Lichter PR, Wright MM, Guire KE et al. Collaborative Initial Glaucoma Treatment Study Group. Perioperative complications of trabeculectomy in the Collaborative Initial Glaucoma Treatment Study (CIGTS). Am J Ophthalmol 2007; 140: 16-22.

17 Gedde SJ, Herndon LW, Brandt JD, Budenz DL, Feuer WJ, Schiffman JC. Surgical complications in the Tube Versus Trabeculectomy Study during the first year of follow-up. Am J Ophthalmol 2007; 143: 23-31.

18 Jonas JB. 1 Ausgabe Biomorphometrie des Nervus Opticus. Enke Verlag: Stuttgart S, Germany, 1989, pp 1-148.

19 Kandori M, Miyazaki D, Yakura K, Komatsu N, Touge C, Ishikura $\mathrm{R}$ et al. Relationship between the number of cytomegalovirus in anterior chamber and severity of anterior segment inflammation. Jpn J Ophthalmol 2013; 57(6): 497-502. 
20 Miyanaga M, Sugita S, Shimizu N, Morio T, Miyata K, Maruyama $\mathrm{K}$ et al. A significant association of viral loads with corneal endothelial cell damage in cytomegalovirus anterior uveitis. Br J Ophthalmol 2010; 94(3): 336-340.

21 Ahuja Y, Ma Khin Pyi S, Malihi M, Hodge DO, Sit AJ. Clinical results of ab interno trabeculotomy using the trabectome for open-angle glaucoma: the Mayo Clinic series in Rochester, Minnesota. Am J Ophthalmol 2013; 156(5): 927-935.

22 Jordan JF, Wecker T, van Oterendorp C, Anton A, Reinhard $\mathrm{T}$, Boehringer $\mathrm{D}$ et al. Trabectome surgery for primary and secondary open angle glaucomas. Graefes Arch Clin Exp Ophthalmol 2013; 251(12): 2753-2760.

23 Ting JL, Damji KF, Stiles MC. Ab interno trabeculectomy: outcomes in exfoliation versus primary open-angle glaucoma. J Cataract Refract Surg 2012; 38(2): 315-323.
24 Klamann MK, Gonnermann J, Maier AK, Bertelmann E, Joussen AM, Torun N. Influence of selective laser trabeculoplasty (SLT) on combined clear cornea phacoemulsification and trabectome outcomes. Graefes Arch Clin Exp Ophthalmol 2014; 252(4): 627-631.

25 Wang Q, Harasymowycz P. Goniopuncture in the treatment of short-term post-trabectome intraocular pressure elevation: a retrospective case series study. J Glaucoma 2013; 22(8): e17-e20.

26 Klamann MK, Gonnermann J, Maier AK, Ruokonen PC, Torun N, Joussen AM et al. Combined clear cornea phacoemulsification in the treatment of pseudoexfoliative glaucoma associated with cataract: significance of trabecular aspiration and ab interno trabeculectomy. Graefes Arch Clin Exp Ophthalmol 2013; 251(9): 2195-2199. 